\title{
CARMENES: high-resolution spectra and precise radial velocities in the red and infrared
}

\author{
A. Quirrenbach, P. J. Amado, I. Ribas, A. Reiners, J. A. \\ Caballero, et al.
}

A. Quirrenbach, P. J. Amado, I. Ribas, A. Reiners, J. A. Caballero, W. Seifert, J. Aceituno, M. Azzaro, D. Baroch, D. Barrado, F. Bauer, S. Becerril, V J S. Bèjar, D. Benítez, M. Brinkmöller, C. Cardona Guillén, C. Cifuentes, J. Colomé, M. Cortés-Contreras, S. Czesla, S. Dreizler, K. Frölich, B. Fuhrmeister, D. Galadí-Enríquez, J. I. González Hernández, R. González Peinado, E. W. Guenther, E. de Guindos, H.-J. Hagen, A. P. Hatzes, P. H. Hauschildt, J. Helmling, Th. Henning, O. Herbort, L. Hernández Guenther, E. de Guindos, H.-J. Hagen, A. P. Hatzes, P. H. Hauschildt, J. Helmling, Th. Henning, O. Herbort, L. Hernández
Castaño, E. Herrero, D. Hintz, S. V. Jeffers, E. N. Johnson, E. de Juan, A. Kaminski, H. Klahr, M. Kürster, M. Lafarga, L. Sairam, M. Lampón, L. M. Lara, R. Launhardt, M. López del Fresno, M. López-Puertas, R. Luque, H. Mandel, E. G. Marfil, E. L. Martín, S. Martín-Ruiz, R. J. Mathar, D. Montes, J. C. Morales, E. Nagel, L. Nortmann, G. Nowak, E. Pallé, V.-M. Passegger, A. Pavlov, S. Pedraz, D. Pérez-Medialdea, M. Perger, R. Rebolo, S. Reffert, E. Rodríguez, C. Rodríguez López, A. Rosich, S. Sabotta, S. Sadegi, M. Salz, A Sánchez-López, J. Sanz-Forcada, P. Sarkis, S. Schäfer, J. Schiller, J. H. M. M. Schmitt, P. Schöfer, A. Schweitzer, D. Shulyak, E. Solano, O. Stahl, M. Tala Pinto, T Trifonov, M. R Zapatero Osorio, F. Yan M. Zechmeister, F. J. Schweitzer, D. Shulyak, E. Solano, O. Stahl, M. Tala Pinto, T. Tronov, M. R. Zapatero Oson, F. Yan, M. Zechneister, F. J. Berdiñas, G. Bergondy, M. Blümcke, C. del Burgo, J. Cano, J. Carro, M. C. Cárdenas, E. Casal, A. Claret, E. Díez-Alonso, M. Doellinger, R. Dorda, C. Feiz, M. Fernández, I. M. Ferro, G. Gaisné, I. Gallardo, M. C. Gálvez-Ortiz, A. García-Piquer, M. L. García-Vargas, R. Garrido, L. Gesa, V. Gómez Galera, E. González-Álvarez, L. González-Cuesta, S. Grohnert, U. Grözinger, J. Guàrdia, A. Guijarro, R. P. Hedrosa, D. Hermann, I. Hermelo, R. Hernández Arabí, F. Hernández Hernando, D. Hidalgo, G. Holgado, A. Huber, K. Huber, P. Huke, M. Kehr, M. Kim, R. Klein, J. Klüter, A. Klutsch, F. Labarga, N. Labiche, A. Lamert, W. Laun, F. J. Lázaro, U. Lemke, R. Lenzen, M. Llamas, J.-L. Lizon, N. Lodieu, M. J. López González, M. López-Morales, J. F. López Salas, J. López-Santiago, H. Magán Madinabeitia, U. Mall, L. Mancini, J. A. Marín Molina, H. Martínez-Rodríguez, D. Maroto Fernández, C. J. Marvin, E. Mirabet, M. E. Moreno-Raya, A. Moya, R. Mundt, V. Naranjo, J. Panduro, J. Pascual, A Pérez-Calpena, M. A. C. Perryman, M. Pluto, A. Ramón, P. Redondo, S. Reinhart, P. Rhode, H.-W. Rix, F. Rodler, R.-R. Rohloff, E. Sánchez-Blanco, M. A. Sánchez Carrasco, L. F. Sarmiento, C. Schmidt, C. Storz, J. B. P. Strachan, J. Stürmer, J. C. Suárez, H M. Tabernero, L. Tal-Or, S. M. Tulloch, R.-G. Ulbrich, G. Veredas, J. L. Vico Linares, M. Vidal-Dasilva, F. Vilardell, K. Wagner, J. Winkler, V. Wolthoff, W. Xu, Z. Zhao, "CARMENES: high-resolution spectra and precise radial velocities in the red and infrared," Proc. SPIE 10702, Ground-based and Airborne Instrumentation for Astronomy VII, 107020 W (27 July 2018); doi: 10.1117/12.2313689 


\section{CARMENES: high-resolution spectra and precise radial velocities in the red and infrared}

A. Quirrenbach ${ }^{\text {a }}$, P. J. Amado ${ }^{\mathrm{b}}$, I. Ribas ${ }^{\mathrm{c}, \mathrm{d}}$, A. Reiners $^{\mathrm{e}}$, J. A. Caballero ${ }^{\mathrm{f}, \mathrm{a}}$, W. Seifert ${ }^{\mathrm{a}}$, J. Aceituno ${ }^{\mathrm{g}}$, M. Azzarog, D. Baroch ${ }^{\mathrm{c}, \mathrm{d}}$, D. Barrado ${ }^{\mathrm{f}}$, F. Bauer ${ }^{\mathrm{b}, \mathrm{e}}$, S. Becerril ${ }^{\mathrm{b}}$, V. J. S. Béjar ${ }^{\mathrm{h}, \mathrm{i}}$,

D. Benítez ${ }^{\mathrm{g}}$, M. Brinkmöller ${ }^{\mathrm{a}}$, C. Cardona Guillén ${ }^{\mathrm{h}, \mathrm{i}}$, C. Cifuentes ${ }^{\mathrm{j}, \mathrm{f}}$, J. Coloméc,d,

M. Cortés-Contreras ${ }^{\mathrm{f}, \mathrm{j}}$, S. Czesla ${ }^{\mathrm{k}}$, S. Dreizlere ${ }^{\mathrm{e}}$, K. Frölich ${ }^{\mathrm{k}}$, B. Fuhrmeister ${ }^{\mathrm{k}}$,

D. Galadí-Enríquez ${ }^{\mathrm{g}}$, J. I. González Hernández,i, ${ }^{\mathrm{h}}$ R. González Peinadoj, E. W. Guenther ${ }^{\mathrm{l}}$, E. de Guindos ${ }^{g}$, H.-J. Hagen ${ }^{\mathrm{k}}$, A. P. Hatzes ${ }^{\text {, }}$ P. H. Hauschildt ${ }^{\mathrm{k}}$, J. Helmling ${ }^{\mathrm{g}}$, Th. Henning ${ }^{\mathrm{m}}$, O. Herbort ${ }^{\mathrm{e}}$, L. Hernández Castaño ${ }^{\mathrm{g}}$, E. Herrero ${ }^{\mathrm{c}, \mathrm{d}}$, D. Hintz ${ }^{\mathrm{k}}$, S. V. Jeffers ${ }^{\mathrm{e}}$, E. N. Johnson ${ }^{\mathrm{e}}$, E. de Juan ${ }^{\mathrm{g}}$, A. Kaminski ${ }^{\mathrm{a}}$, H. Klahr ${ }^{\mathrm{m}}$, M. Kürster ${ }^{\mathrm{m}}$, M. Lafarga ${ }^{\mathrm{c}, \mathrm{d}}$, L. Sairam ${ }^{\mathrm{k}}$, M. Lampón ${ }^{\mathrm{b}}$, L. M. Lara ${ }^{\mathrm{b}}$, R. Launhardt ${ }^{\mathrm{m}}$, M. López del Fresno ${ }^{\mathrm{f}}$, M. López-Puertas ${ }^{\mathrm{b}}$, R. Luque ${ }^{\mathrm{h}, \mathrm{i}, \mathrm{a}}$,

H. Mandel ${ }^{a}$, E. G. Marfili , E. L. Martín ${ }^{\mathrm{f}}$, S. Martín-Ruiz ${ }^{\mathrm{b}}$, R. J. Mathar ${ }^{\mathrm{m}}$, D. Montes ${ }^{\mathrm{j}}$, J. C. Morales ${ }^{\mathrm{c}, \mathrm{d}}$, E. Nagel ${ }^{\mathrm{k}}$, L. Nortmann ${ }^{\mathrm{h}, \mathrm{i}}$, G. Nowak ${ }^{\mathrm{h}, \mathrm{i}}$, E. Palléh,i, V. M. Passegger ${ }^{\mathrm{k}, \mathrm{e}}$, A. Pavlov ${ }^{\mathrm{m}}$, S. Pedraz ${ }^{\mathrm{g}}$, D. Pérez-Medialdea ${ }^{\mathrm{b}}$, M. Perger ${ }^{\mathrm{c}, \mathrm{d}}$, R. Rebolo, ${ }^{\mathrm{h}, \mathrm{i}}$, S. Reffert ${ }^{\mathrm{a}}$, E. Rodríguez ${ }^{b}$ C. Rodríguez López ${ }^{b}$, A. Rosich ${ }^{c, d}$, S. Sabotta ${ }^{1}$, S. Sadegia ${ }^{a}$, M. Salz ${ }^{k}$, A. Sánchez-López ${ }^{b}$, J. Sanz-Forcada ${ }^{\mathrm{f}}$, P. Sarkis ${ }^{\mathrm{m}}$, S. Schäfer ${ }^{\mathrm{e}}$, J. Schiller ${ }^{\mathrm{l}}$, J. H. M. M. Schmitt ${ }^{\mathrm{k}}$, P. Schöfer ${ }^{\mathrm{e}}$, A. Schweitzerk ${ }^{\mathrm{k}}$, D. Shulyak ${ }^{\mathrm{e}}$, E. Solano ${ }^{\mathrm{f}}$, O. Stahl ${ }^{\mathrm{a}}$, M. Tala Pinto ${ }^{\mathrm{a}}$, T. Trifonov ${ }^{\mathrm{m}}$, M. R. Zapatero Osorio ${ }^{\mathrm{f}}$, F. Yan ${ }^{\mathrm{m}}$, M. Zechmeister ${ }^{\mathrm{e}}$, F. J. Abellán ${ }^{\mathrm{n}, \mathrm{j}}$, M. Abril ${ }^{\mathrm{b}}$, F. J. Alonso-Floriano ${ }^{\mathrm{o} j}$, M. Ammler-von Eiffp,l, G. Anglada-Escudéq, ${ }^{\mathrm{q}, \mathrm{b}}$, H. Anwand-Heerwarte,

B. Arroyo-Torres ${ }^{\mathrm{g}}$, Z. M. Berdiñas ${ }^{\mathrm{r}, \mathrm{b}}$, G. Bergond ${ }^{\mathrm{g}}$, M. Blümckel, C. del Burgo ${ }^{\mathrm{s}}$, J. Cano ${ }^{\mathrm{j}}$, J. Carro ${ }^{j}$, M. C. Cárdenas ${ }^{\mathrm{m}}$, E. Casal ${ }^{\mathrm{b}}$, A. Claret ${ }^{\mathrm{b}}$, E. Díez-Alonso ${ }^{\mathrm{t}, \mathrm{j}}$, M. Doellinger ${ }^{\mathrm{l}}$, R. Dorda ${ }^{\mathrm{u}, \mathrm{j}}$, C. Feiz ${ }^{\mathrm{a}}$, M. Fernández ${ }^{\mathrm{b}}$, I. M. Ferro ${ }^{\mathrm{b}}$, G. Gaisné ${ }^{\mathrm{h}, \mathrm{i}}$, I. Gallardo M. C. Gálvez-Ortiz ${ }^{\mathrm{f}}$, A. Garcia-Piquer, ${ }^{\mathrm{c}, \mathrm{d}}$, M. L. García-Vargas ${ }^{\mathrm{v}}$, R. Garrido ${ }^{\mathrm{b}}$, L. Gesa ${ }^{\mathrm{c}, \mathrm{d}}$, V. Gómez Galera ${ }^{g}$, E. González-Álvarez ${ }^{\mathrm{w}, \mathrm{j}}$, L. González-Cuesta ${ }^{\mathrm{h}, \mathrm{i}}$, S. Grohnert ${ }^{\mathrm{a}}$, U. Grözinger ${ }^{\mathrm{m}}$, J. Guàrdia ${ }^{\mathrm{c}, \mathrm{d}}$, A. Guijarro ${ }^{\mathrm{g}}$, R. P. Hedrosa ${ }^{\mathrm{g}}$, D. Hermann ${ }^{\mathrm{m}}$, I. Hermelo ${ }^{\mathrm{g}}$, R. Hernández Arabíg, F. Hernández Hernandog, D. Hidalgo ${ }^{\text {h,i,j, }}$, G. Holgado ${ }^{\mathrm{h}, \mathrm{i}, \mathrm{j}}$, A. Huber ${ }^{\mathrm{m}}$, K. F. Huber ${ }^{\mathrm{k}}$, P. Huke ${ }^{\mathrm{e}}$, M. Kehrr, M. Kim ${ }^{\mathrm{x}, \mathrm{a}}$, R. Klein ${ }^{\mathrm{m}}$, J. Klüter ${ }^{\mathrm{y}, \mathrm{a}}$, A. Klutsch ${ }^{\mathrm{z}, \mathrm{j}}$,

F. Labarga ${ }^{j}$, N. Labiche ${ }^{\mathrm{a}}$, A. Lamerte, W. Laun ${ }^{\mathrm{m}}$, F. J. Lázaro ${ }^{\mathrm{j}}$, U. Lemke ${ }^{\mathrm{e}}$, R. Lenzen ${ }^{\mathrm{m}}$,

M. Llamas ${ }^{\mathrm{j}}$, J.-L. Lizon ${ }^{\text {aa }}$, N. Lodieu, ${ }^{\mathrm{h}, \mathrm{i}}$, M. J. López González ${ }^{\mathrm{b}}$, M. López-Morales ${ }^{\mathrm{ab}}$, J.F. López Salas ${ }^{\mathrm{g}}$, J. López-Santiago ${ }^{\mathrm{ac}, \mathrm{j}}$, H. Magán Madinabeitia ${ }^{\mathrm{b}}$, U. Mall ${ }^{\mathrm{m}}$, L. Manciniad,m, J. A. Marín Molina ${ }^{\mathrm{g}}$, H. Martínez-Rodríguez ${ }^{\mathrm{a}, \mathrm{j}}$, D. Maroto Fernández ${ }^{\mathrm{g}}$, C. J. Marvine ${ }^{\mathrm{e}}$ E. Mirabet ${ }^{\mathrm{b}}$, M. E. Moreno-Raya ${ }^{\mathrm{g}}$, A. Moya ${ }^{\mathrm{af}, \mathrm{f}}$, R. Mundt ${ }^{\mathrm{m}}$, V. Naranjo ${ }^{\mathrm{m}}$, A. Ofir $^{\mathrm{ag}, \mathrm{e}}$,

J. Panduro ${ }^{\mathrm{m}}$, J. Pascual ${ }^{\mathrm{g}}$, A. Pérez-Calpena ${ }^{\mathrm{v}}$, M. A. C. Perryman ${ }^{\text {ah }}$, M. Pluto ${ }^{\mathrm{l}}$, A. Ramón ${ }^{\mathrm{b}}$, P. Redondo ${ }^{\text {h,i }}$, S. Reinhart ${ }^{\mathrm{g}}$, P. Rhode ${ }^{\mathrm{e}}$, H.-W. Rix ${ }^{\mathrm{m}}$, F. Rodler $^{\mathrm{a}, \mathrm{e}}$, R.-R. Rohloff ${ }^{\mathrm{m}}$,

E. Sánchez-Blanco ${ }^{\mathrm{aj}}$, M. A. Sánchez Carrasco ${ }^{\mathrm{b}}$, L. F. Sarmiento ${ }^{\mathrm{e}}$, C. Schmidte ${ }^{\mathrm{e}}$, C. Storz ${ }^{\mathrm{m}}$, J. B. P. Strachan ${ }^{q}$, J. Stürmerak,a, J. C. Suárez ${ }^{\mathrm{al,b}}$, H. M. Tabernero ${ }^{\mathrm{u}, \mathrm{j}}$, L. Tal-Or ${ }^{\mathrm{am}, \mathrm{e}}$,

S. M. Tulloch ${ }^{y}$, R.-G. Ulbrich ${ }^{\mathrm{e}}$, G. Veredas ${ }^{\mathrm{a}}$, J. I. Vico Linares ${ }^{\mathrm{g}}$, M. Vidal-Dasilva ${ }^{\mathrm{f}}$, F. Vilardell ${ }^{\text {c,d }}$, K. Wagner ${ }^{\mathrm{a}}$, J. Winkler ${ }^{\mathrm{l}}$, V. Wolthoff ${ }^{\mathrm{a}}, \mathrm{W} . \mathrm{Xu}^{\text {an,a }}$, and Z. Zhao ${ }^{\mathrm{e}}$

${ }^{a}$ Landessternwarte, Zentrum für Astronomie der Universität Heidelberg, Königstuhl 12, D-69117 Heidelberg, Germany

bInstituto de Astrofísica de Andalucía (CSIC), Glorieta de la Astronomía s/n, E-18008 Granada, Spain

Ground-based and Airborne Instrumentation for Astronomy VII, edited by Christopher J. Evans, Luc Simard, Hideki Takami, Proc. of SPIE Vol. 10702, 107020W · C 2018 SPIE · CCC code: 0277-786X/18/\$18 · doi: 10.1117/12.2313689 
'Institut de Ciències de l'Espai (ICE, CSIC), Campus UAB, c/ de Can Magrans s/n, E-08193 Bellaterra, Barcelona, Spain

${ }^{d}$ Institut d'Estudis Espacials de Catalunya (IEEC), E-08034 Barcelona, Spain eInstitut für Astrophysik, Georg-August-Universität Göttingen, Friedrich-Hund-Platz 1, D-37077 Göttingen, Germany

${ }^{\mathrm{f}}$ Centro de Astrobiología (CSIC-INTA), Campus ESAC, Camino Bajo del Castillo s/n, E-28692 Villanueva de la Cañada, Madrid, Spain

${ }^{g}$ Centro Astronómico Hispano-Alemán (MPG-CSIC), Observatorio Astronómico de Calar Alto, Sierra de los Filabres, E-04550 Gérgal, Almería, Spain

hInstituto de Astrofísica de Canarias, c/ Vía Láctea s/n, E-38205 La Laguna, Tenerife, Spain

iDepartamento de Astrofísica, Universidad de La Laguna, E-38206 Tenerife, Spain

${ }^{j}$ Departamento de Física de la Tierra y Astrofísica \& UPARCOS-UCM, Facultad de Física, Universidad Complutense de Madrid, E-28040 Madrid, Spain

${ }^{k}$ Hamburger Sternwarte, Gojenbergsweg 112, D-21029 Hamburg, Germany

${ }^{1}$ Thüringer Landessternwarte Tautenburg, Sternwarte 5, D-07778 Tautenburg, Germany

${ }^{m}$ Max-Planck-Institut für Astronomie, Königstuhl 17, D-69117 Heidelberg, Germany

${ }^{\mathrm{n}}$ Departamento de Astronomía y Astrofísica, Universidad de Valencia, c/ Dr. Moliner 50, E-46100 Burjassot, Spain

${ }^{\circ}$ Leiden Observatory, Leiden University, Postbus 9513, 2300 RA Leiden, The Netherlands

pMax-Planck-Institut für Sonnensystemforschung, Justus-von-Liebig-Weg 3, D-37077 Göttingen, Germany

${ }^{\mathrm{q}}$ School of Physics and Astronomy, Queen Mary, University of London, 327 Mile End Road, London, E1 4NS, UK

${ }^{r}$ Departamento de Astronomía, Universidad de Chile, Camino del Observatorio, 1515 Las Condes, Santiago, Chile

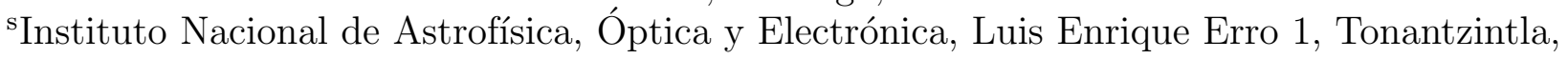
72840 Puebla, Mexico

${ }^{t}$ Department of Exploitation and Exploration of Mines, University of Oviedo, E-33004 Oviedo, Spain

uDepartamento de Física, Ingeniería de Sistemas y Teoría de la Señal, Universidad de Alicante,

Carretera de San Vicente s/n, San Vicente del Raspeig, E-03690 Alicante, Spain

vFRACTAL SLNE, c/ Tulipán 2, p. 13, 1A, E-28231 Las Rozas, Madrid, Spain

wINAF - Osservatorio Astronomico di Palermo, Piazza Parlamento 1, I-90134 Palermo, Italy 'Institut für Theoretische Physik und Astrophysik, Christian-Albrechts-Universität zu Kiel, Leibnizstrasse 15, D-24118 Kiel, Germany

${ }^{y}$ Zentrum für Astronomie der Universität Heidelberg, Astronomisches Rechen-Institut, Mönchhofstr. 12, D-69120 Heidelberg, Germany

${ }^{2}$ INAF - Osservatorio Astrofisico di Catania, via S. Sofia 78, I-95123 Catania, Italy

${ }^{a a}$ European Organisation for Astronomical Research in the Southern Hemisphere,

Karl-Schwarzschild-Str. 2, D-85748 Garching bei München, Germany

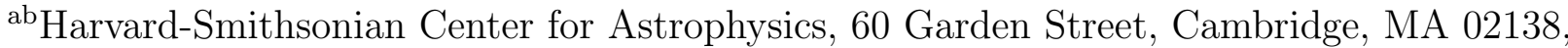
USA

${ }^{a c}$ Departamento de Teoría de la Señal y Comunicaciones, Edificio Torres Quevedo, Escuela Politécnica Superior, Universidad Carlos III de Madrid, Av. de la Universidad 30, E-28911 Leganés, Madrid 


\author{
${ }^{a d}$ Dipartimento di Fisica, Universitá di Roma Tor Vergata, Via della Ricerca Scientifica 1, \\ I-00133, Roma, Italy \\ ${ }^{a e}$ Department of Physics and Astronomy and Pittsburgh Particle Physics, Astrophysics and \\ Cosmology Center (PITT PACC), University of Pittsburgh, 3941 O'Hara Street, Pittsburgh, \\ PA 15260, USA

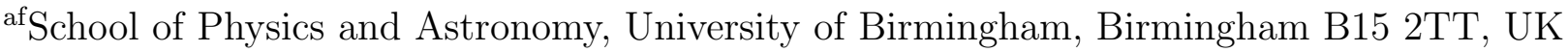

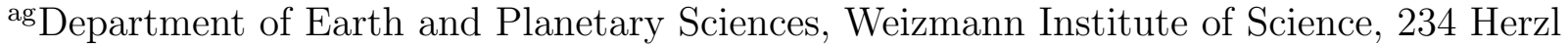 \\ Street, 76100 Rehovot, Israel \\ ${ }^{\text {ah }}$ School of Physics, University College Dublin, Belfield, Dublin 4, Ireland \\ ai European Organisation for Astronomical Research in the Southern Hemisphere, Alonso de \\ Córdova 3107, Vitacura, Santiago de Chile, Chile \\ ajOptical Development, Avenida Pablo Iglesias 7, local 5, E-41008 Sevilla, Spain \\ ${ }^{a k}$ Department of Astronomy and Astrophysics, University of Chicago, 5640 S. Ellis Avenue, \\ 60637 Chicago, IL, USA \\ ${ }^{a l}$ Universidad de Granada, Departamento de Física Teórica y de Cosmos, Fuentenueva \\ Campus, E-18007, Granada, Spain \\ ${ }^{a m}$ School of Physics and Astronomy, Tel Aviv University, Tel Aviv 69978, Israel \\ ${ }^{a n}$ Wenli Xu Optical System Engineering, Kirchenstr. 6, D-74937 Spechbach, Germany
}

\begin{abstract}
The CARMENES instrument has been operational at the $3.5 \mathrm{~m}$ telescope of Calar Alto Observatory since January 2016. It consists of two cross-dispersed échelle spectrographs covering the wavelength range from 0.52 to $1.71 \mu \mathrm{m}$. CARMENES is currently conducting a radial-velocity survey of more than $300 \mathrm{M}$ dwarfs, with a sensitivity sufficient to detect terrestrial planets in their habitable zones. This survey has already yielded a comprehensive spectral atlas of $324 \mathrm{M}$ dwarfs, and it is providing a wealth of diagnostic information on activity in cool stars. The CARMENES Survey data have confirmed a number of known M star planets, and revealed previously unknown planets of GJ 15 A, GJ 1148, and GJ 617 A. CARMENES data have also been used to determine the mass of the transiting planet K2-18 b, and to measure atomic and molecular absorption in planetary atmospheres through transit spectroscopy.
\end{abstract}

Keywords: Spectrographs, Optical Instrumentation, Near-Infrared Instrumentation, Extrasolar Planets, Cool Stars, M Dwarfs

\title{
1. INTRODUCTION
}

The CARMENES instrument is a pair of high-resolution spectrographs optimized for measuring radial velocities in the wavelength range from 0.52 to $1.71 \mu \mathrm{m}$; it saw first light at Calar Alto Observatory in November 2015 and started regular observing in January 2016. The first two years of regular operation were mostly dedicated to a survey of $\sim 340 \mathrm{M}$ dwarf stars. After several improvements of the operational procedures and of the calibration, we have established the capability of the instrument to obtain $\mathrm{m} \mathrm{s}^{-1}$ precision radial velocities. In the following sections we provide a brief overview of the instrument, discuss its performance, and present a few examples of early scientific results from the survey.

More detailed accounts on the design and construction of CARMENES have been given at previous SPIE conferences (see Quirrenbach et al. 2010, 2012, 2014, 2016, and references therein); current information on CARMENES can be found at http://carmenes.caha.es.

Further author information: E-mail: A.Quirrenbach@lsw.uni-heidelberg.de, Telephone: +49 6221541792 


\section{THE CARMENES INSTRUMENT}

\subsection{Instrument Overview}

The CARMENES instrument has been built specifically for the purpose of obtaining precise radial velocity measurements of cool low-mass stars. The front end of CARMENES is attached to the Cassegrain focus of the Calar Alto $3.5 \mathrm{~m}$ telescope. Here the light is separated into the visible and near-IR channels by a dichroic beam splitter centered at $0.96 \mu \mathrm{m}$. The two spectral ranges are coupled with fibers into two separate spectrographs, which are mounted on optical benches inside vacuum tanks, which are located in the former coudé laboratory of the $3.5 \mathrm{~m}$ dome. The main instrument sub-systems of CARMENES are the following:

\subsection{Front End}

The front end, which is attached to the Cassegrain focus of the telescope, contains the acquisition and guiding camera, an atmospheric dispersion compensator consisting of two counter-rotating prism pairs, the dichroic beam splitter, a shutter (only in the visible channel), movable mirrors to switch between the sky and calibration light, and the fiber heads (Seifert et al. 2012). The first mirror in the front end is motorized; when it is moved out of the beam the light passes straight through to a separate focus so that it is possible to switch rapidly between CARMENES and another Cassegrain instrument (currently the integral-field spectrograph PMAS).

\subsection{Fiber Link}

The optical fibers coupling the front end to the spectrographs also accomplish the vital task of "scrambling", i.e., of reducing the jitter of the stellar image at the spectrograph "pseudo-slit" entrance with respect to guiding errors and to seeing fluctuations at the fiber input. Long $(42 \mathrm{~m}$ and $50 \mathrm{~m}$ for the visible-light and near-infrared arms, respectively) circular fibers leading from the telescope to the coudé room are connected there to shorter $(10 \mathrm{~m})$ fiber sections with an octagonal cross section. This facilitates replacement of the long fibers in case they should be damaged, and ensures very good scrambling properties (Stürmer et al. 2014). The fiber diameter has been chosen to provide a 1.'5 diameter acceptance angle on the sky, corresponding to somewhat worse than median seeing on Calar Alto.

\subsection{Visible-Light Spectrograph}

The visible-light spectrograph of CARMENES is a grism cross-dispersed, white pupil, échelle spectrograph working in quasi-Littrow mode using a two-beam, two-slice, image slicer (Seifert et al. 2016). It covers the wavelength range from $0.52 \mu \mathrm{m}$ to $1.05 \mu \mathrm{m}$ with a resolving power of $R=94,600$. The mean sampling is 2.8 pixels per resolution element, but it varies strongly along each order, as is typical for an R4 échelle. The spectrograph accepts light from two fibers; the first fiber carries the light from the target star, while the second fiber can either be used for simultaneous wavelength calibration or for monitoring the sky emission. The spectrograph is housed in a vacuum vessel and operated at room temperature; it follows the ambient temperature with a long time constant. The detector is a back-side illuminated $4112 \times 4096$ pixel CCD with a gradient coating (model e2v CCD231-84).

\subsection{Near-Infrared Spectrograph}

The optical design and mechanical layout of the near-infrared spectrograph are very similar to those of its visible-light counterpart. It provides a spectral resolution $R=80,400$ over the wavelength range from $0.95 \mu \mathrm{m}$ to $1.71 \mu \mathrm{m}$ with a mean sampling of 2.5 pixels per resolution element. The near-IR spectrograph is actively cooled to $140 \mathrm{~K}$ with a continuous flow of gaseous nitrogen, as required to keep the thermal background below the detector dark current. The spectrograph employs a mosaic of two $2048 \times 2048$ pixel HAWAII-2RG infrared arrays with a long-wavelength cutoff at $2.5 \mu \mathrm{m}$ (Amado et al. 2012). The near-IR cooling system uses an external heat exchanger / gas evaporator unit, which is fed by liquid nitrogen and provides a continuous flow of gaseous nitrogen (Becerril et al. 2012). 

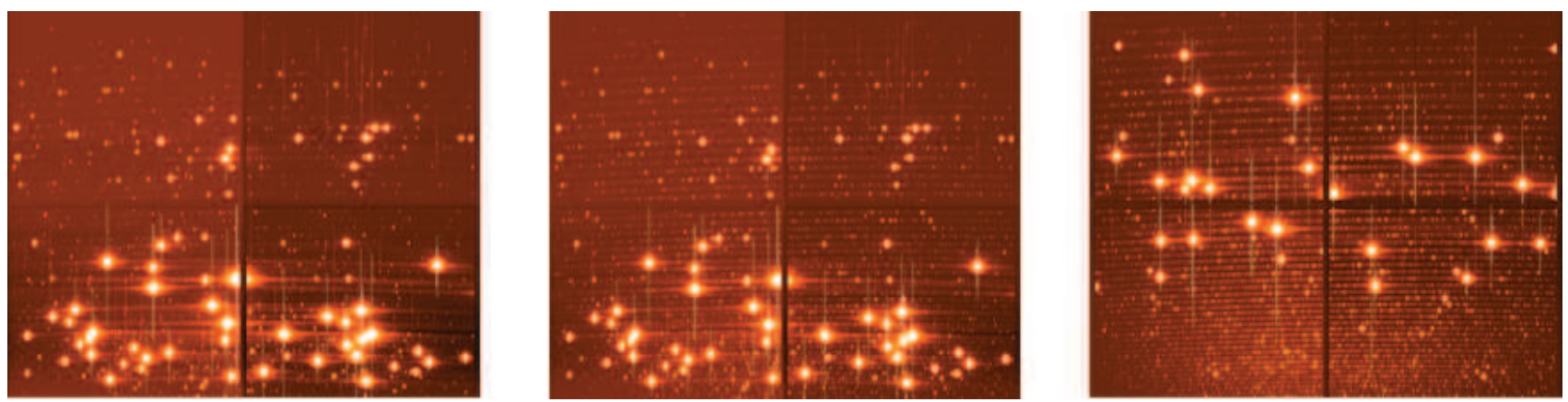

Figure 1. Hollow-cathode calibration lamp exposures (from left to right: Th-Ne, U-Ne, and U-Ar) taken with the visiblelight spectrograph. The spectra of the lamps provide rich calibration information, but very bright noble gas lines prevent their use for simultaneous calibration of stellar spectra.

\subsection{Exposure Meters}

The zeroth-order light from the échelle grating in each of the two spectrographs is reflected back to a photomultiplier tube, which records the received intensity with a high sampling rate (Seifert et al. 2012). This information is used by the data reduction pipeline for an accurate barycentric correction, referencing the observed radial velocities to an inertial system. It can also be used to make real-time adjustments to the integration time depending on atmospheric conditions.

\subsection{Calibration Units and Wavelength Calibration}

Both CARMENES spectrographs use hollow-cathode emission line lamps and Fabry-Pérot etalons for spectral calibration (Schäfer et al. 2018). For each spectrograph, the arc lamps as well as quartz lamps for flat-fielding are housed in a calibration unit that is connected to the front end with a fiber link.

Precision radial-velocity measurements at red optical and infrared wavelengths require a sophisticated strategy for wavelength calibration. In a spectrograph such as HARPS, which works in the blue part of the visible wavelength range, Th-Ar hollow-cathode lamps (HCLs) provide a dense forest of emission lines that are very well suited for wavelength calibration because they are not severely affected by noble gas emission (Ar). In contrast, CARMENES operates in a region where all HCL fill gases emit very bright lines (see Fig. 1). A fair number of these lines are much stronger than the Th (or other cathode material) lines and saturate the detectors for any integration time long enough to expose the Th spectrum well. Furthermore, Th emits most of its lines at optical wavelengths, but does not have a similarly rich spectrum in the near infrared. During the construction phase of CARMENES, we investigated different types of HCLs and constructed new line lists (Sarmiento et al. 2014). For the calibration of CARMENES, we are using three different types of emission lamps to provide optimal coverage over the whole wavelength range: Th-Ne, U-Ar, and U-Ne, see Fig. 1.

Because of the bright noble gas lines, the HCLs are not suitable for simultaneous calibration of stellar spectra. We therefore incorporated in the instrument two thermally stabilized Fabry-Pérot etalons (FPs) covering the entire CARMENES wavelength range with dense emission lines. With more than $10^{4} \mathrm{FP}$ emission lines, we can construct a precise wavelength solution from the FP comb that is incorporated in our wavelength calibration scheme (Bauer et al. 2015). The FPs are also used during the night to monitor short-term spectrograph drifts; long-term stability is ensured by comparing the FPs to HCL exposures taken during daytime before and after the observations.

\subsection{Instrument Control System}

The coordination of the observations and the management of the sub-systems of CARMENES is handled by the instrument control system (ICS), which provides a graphical user interface and tools to operate the instrument in a safe and integrated manner (Colomé et al. 2016). The ICS includes a scheduling algorithm that can autonomously prioritize and select targets for observation from an input catalog containing all stars in the survey sample, along with information on the desired observing cadence for each one of them (Garcia-Piquer et al. 2017). 


\subsection{Infrastructure}

The two CARMENES spectrographs and their ancillary equipment (electronics, pumps, nitrogen supply, etc.) are located in the former coudé room of the $3.5 \mathrm{~m}$ telescope dome, one floor below the telescope. Each spectrograph is placed within its own temperature-controlled chamber, which provides shielding from annual temperature variations and from heat sources. A computer-controlled interlock system monitors the status of the instrument and of the auxiliary systems, displays information about their overall status and health, and issues alarms in case of a malfunction (Helmling et al. 2016).

\subsection{Data Reduction Pipeline}

For standard radial-velocity observations, each CARMENES spectrograph simultaneously receives light from the target star in the first (science) fiber and from the corresponding Fabry-Pérot etalon in the second (calibration) fiber. For faint targets, the second fiber can alternatively be used as a sky fiber. The data reduction pipeline CARACAL (Caballero et al. 2016b) applies standard dark and bias corrections, traces the échelle orders, performs a flat-optimal extraction of the spectra (Zechmeister et al. 2014), and provides the wavelength calibration, leading to fully reduced visible-light and near-infrared spectra.

The computation of the radial velocities is carried out by a second pipeline, SERVAL (Zechmeister et al. 2018). SERVAL uses an iterative approach for the construction of the reference template. The algorithm first derives approximate RVs measured against an observed spectrum, and then improves the template by co-adding all observed spectra and recomputing the RVs. SERVAL yields not only an output consisting of time series of high precision RVs, but also of a number of spectral line indicators that can be useful diagnostic tools for stellar variability. In addition, SERVAL computes indicators of the line width and of the wavelength-dependence of the RV for each observation (see Sect. 5.2).

\section{THE CARMENES SURVEY}

\subsection{Scientific Preparation of the CARMENS Survey}

To optimize the scientific return of CARMENES, we performed a careful analysis of the targets prior to the assembly of the input catalog. A number of critical stellar properties were collected for each individual target candidate, including effective temperature and spectral type, near-IR and visible photometry, distance, projected rotational velocity, and main activity indicators ( $\mathrm{H} \alpha$ and X-ray luminosity). In addition, we also tabulated noncritical information for as many sample candidates as possible, including kinematics (proper motions, absolute radial velocities, Galactocentric space velocities), element abundances, ages, variability (photometry and spectroscopy), multiplicity (from spectroscopy and imaging), and secondary activity indicators.

We started by assembling this information from the literature, and conducted a dedicated observing program (high- and low-resolution spectroscopy, high- and low-resolution imaging) to obtain these parameters in a systematic way. From our low-resolution spectroscopy, carried out with CAFOS at the Calar Alto $2.2 \mathrm{~m}$ telescope, we derived spectral classifications for 753 stars and found that previous spectral type estimates from photometry were systematically too late for many stars (Alonso-Floriano et al. 2015). From the same data, we also determined gravity, metallicity and activity indices.

High-resolution I-band images of 490 candidate target stars were obtained with the lucky imaging camera FastCam at the $1.5 \mathrm{~m}$ Telescopio Carlos Sánchez at the Observatorio del Teide (Cortés-Contreras et al. 2017). In these, we identified 80 bound companions, 30 of them previously unknown. This information on the binary fraction of M dwarfs is interesting in itself, and it helped eliminate unsuitable stars from the CARMENES Survey sample, as contamination from a secondary spectrum would severely hamper the determination of precise radial velocities.

We gathered a total of 1734 high-resolution spectra of $480 \mathrm{M}$ dwarfs with the CAFE (Calar Alto), FEROS (ESO La Silla), and HRS (Hobby-Eberly-Telescope) Spectrographs (Jeffers et al. 2018). These were used to

determine rotational velocities $(v \sin i)$, to analyze indicators of magnetic activity $\left(\mathrm{H} \alpha, R_{\mathrm{HK}}^{\prime}\right)$ for individual stars, and to investigate statistical relations of these quantities with each other and with other stellar properties. 


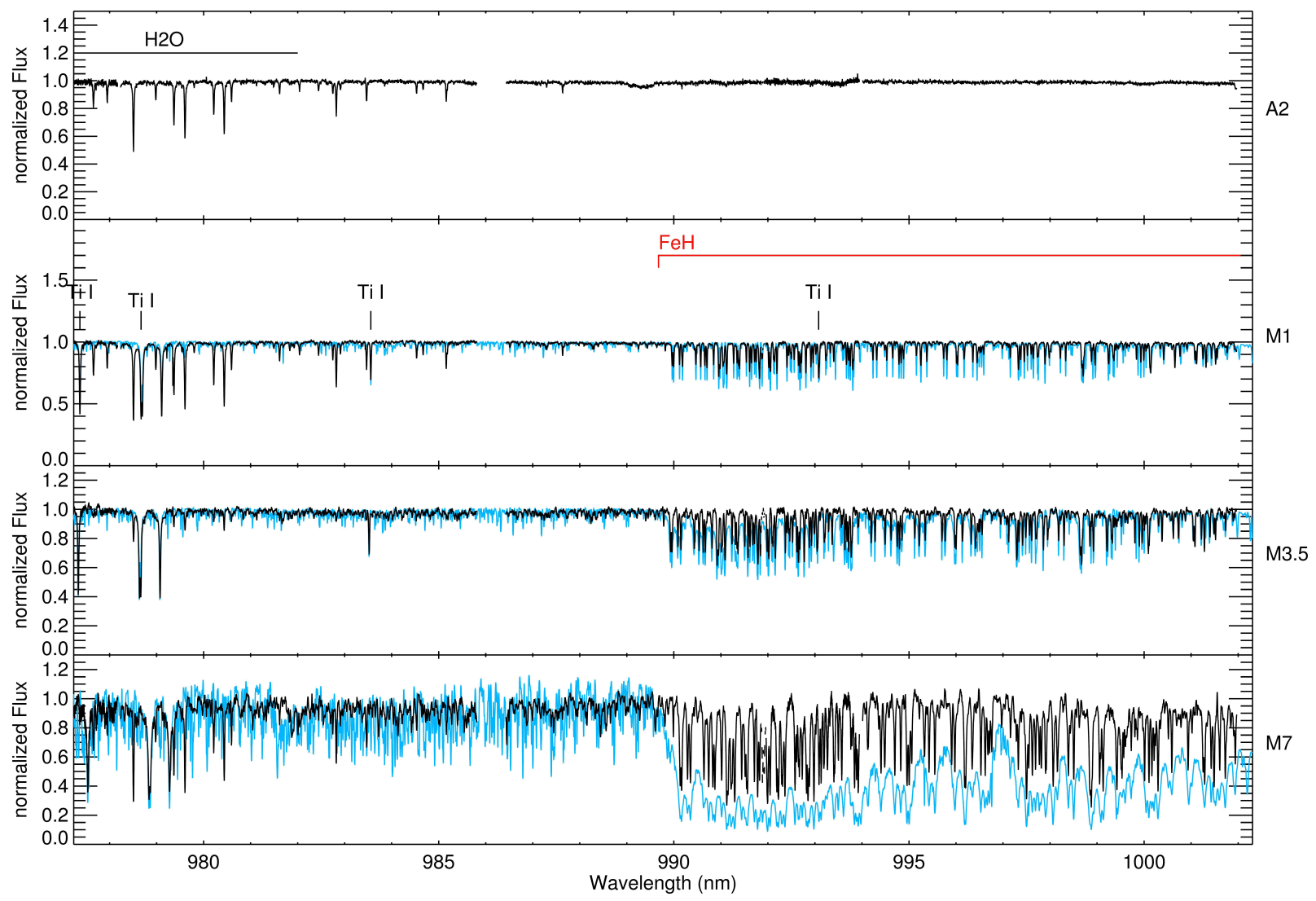

Figure 2. Section of CARMENES-NIR spectra (black) in the wavelength region around $1 \mu \mathrm{m}$. From top to bottom: telluric standard, and examples of early M (GX And), mid M (Luyten's Star), and late M (Teegarden's Star) stars. The light blue lines represent PHOENIX model atmospheres (Husser et al. 2013). These models over-predict the depth of the FeH band beginning at $990 \mathrm{~nm}$ marked in red. Adopted from Reiners et al. (2018b).

\subsection{The CARMENES Survey Sample}

The information described in the previous section has been incorporated into Carmencita, the CARMEN[ES] Cool star Information and daTa Archive, which catalogs over 2000 carefully-selected M dwarfs at $\delta \geq-23^{\circ}$ (Caballero et al. 2016a). This data base is perhaps the most comprehensive source of data on bright, nearby, M dwarfs; it formed the starting point for the compilation of the CARMENES Survey sample. To define this survey sample, we eliminated binaries with separation $<5^{\prime \prime}$, and ranked the remaining CARMENCITA M dwarfs by J-band apparent magnitude within each spectral subtype. We then selected the brightest stars in each subclass, thus creating a sliding magnitude cut-off that helps including stars with later spectral subtypes, while maintaining a simple selection criterion that can be modeled easily in statistical analyses. We thus obtained a sample that covers the M spectral range as uniformly as possible, and that also takes advantage of the "sweet spot" for CARMENES, which is the M3 V to M4V spectral range: Earlier M subtypes can be observed quite efficiently with spectrographs working at bluer wavelengths, whereas on the other hand reaching larger numbers of late M stars requires near-IR spectrographs at larger telescopes. The CARMENES Sample is described in more detail in Quirrenbach et al. (2016) and Reiners et al. (2018b).

\section{M DWARFS IN THE CARMENES SURVEY}

Due to its large wavelength coverage, the CARMENES Survey is generating a unique data set for studies of M star atmospheres, rotation, and activity. The spectra cover important diagnostic lines for activity (e.g., $\mathrm{H} \alpha$, the Na I D1 and D2 lines, the Ca II infrared triplet, He I $\lambda 10833 \AA$ ), as well as FeH bands, from which the 
magnetic field can be inferred. Correlating the time series of these features with each other, and with wavelengthdependent radial velocities, provides excellent handles for the discrimination between planetary companions and stellar radial velocity jitter. These data are also generating new insight into the physical properties of $\mathrm{M}$ dwarf atmospheres, and the impact of activity and flares on the habitability of $\mathrm{M}$ star planets. A small section around $1 \mu \mathrm{m}$ of four CARMENES near-infrared sample spectra is shown in Fig. 2.

\subsection{Stellar Parameters}

The spectra from the CARMENES Survey are well suited for determining stellar parameters, due to their high resolution and signal-to-noise. Passegger et al. (2018) used CARMENES spectra of $235 \mathrm{M}$ dwarfs, along with spectra from other instruments for 65 additional stars, to determine their effective temperatures, gravities, and metallicities. They used the latest PHOENIX-ACES models (Husser et al. 2013) and found that they fit the observations well. This characterization of the target stars provides the context for statistical analyses of the planets detected with CARMENES and other future surveys.

Several M dwarfs in the CARMENES Survey sample are members of physical binaries with FGK primaries. These systems can be used to help calibrate $\mathrm{M}$ star metallicities, as this quantity is much easier to determine for the earlier-type primaries (Montes et al. 2018).

\subsection{Stellar Activity}

The atmospheres of M dwarfs display a variety of dynamical phenomena, which can be investigated with highresolution spectra of these stars. Inhomogeneities of the photosphere such as spots, which co-rotate with the star, cause periodic variations of line profiles and measured radial velocities. Strong magnetic fields lead to Zeeman broadening of the lines, and suppress convective blueshift, leading to a net redshift of the spectrum. Chromospheric activity is most apparent in the $\mathrm{H} \alpha$ line, giving rise to the classification of "active" ( $\mathrm{H} \alpha$ in emission) and "non-active" ( $\mathrm{H} \alpha$ in absorption) $\mathrm{M}$ dwarfs.

The CARMENES Survey is generating a unique treasure trove for detailed studies of M star activity, because it combines high spectral resolution with large wavelength coverage and temporal sampling (dozens of spectra for each star). The target list contains both active and non-active stars covering the range from M0 to M9 (with almost all stars of type M5 or later being active; Reiners et al. 2018b), making it possible to correlate the incidence of signatures of activity with spectral type.

Radial-velocity variations with a scatter of $\sigma_{\mathrm{RV}}>10 \mathrm{~m} \mathrm{~s}^{-1}$ in stars with $v \sin i>2 \mathrm{~km} \mathrm{~s}^{-1}$ are a hallmark of activity (Tal-Or et al. 2018). Such "active RV-loud" stars are much more common among the later spectral sub-types, comprising more than half of the CARMENES sample at M6 and later. The radial-velocity scatter is correlated with the rotation rate; in many cases the RV amplitude decreases with increasing wavelength.

Chromospheric activity gives rise to complex and variable $\mathrm{H} \alpha$ line profiles. $\mathrm{H} \alpha$ line wing asymmetries are rather common among the most active $\mathrm{M}$ dwarfs in the CARMENES sample; they can be interpreted as an indicator of coronal rain (Fuhrmeister et al. 2018). This phenomenon has been described for coronal loops in the Sun, where catastrophic cooling causes plasma to rain down from chromospheric heights. Further observations with high cadence, and ideally encompassing higher Balmer lines (which are not covered by CARMENES), would help to clarify this picture.

\subsection{Spectroscopic Binaries}

Not surprisingly, the CARMENES sample contains a number of previously unknown binary stars. Of particular interest among these are the double-lined spectroscopic binaries (SB2s), because an analysis of both line systems yields the mass ratio (from the ratio of the two RV curve amplitudes), and the two spectral types. Baroch et al. (2018, submitted to A\&A) have derived these parameters for nine new SB2s discovered with CARMENES; they find that the mass ratios are on average smaller than those of more massive binaries. The Gaia mission will yield the inclinations of these systems, which is the only parameter missing for a dynamical measurement of the masses of both components. The CARMENES binaries will thus become important cornerstones for the M dwarf mass calibration. 


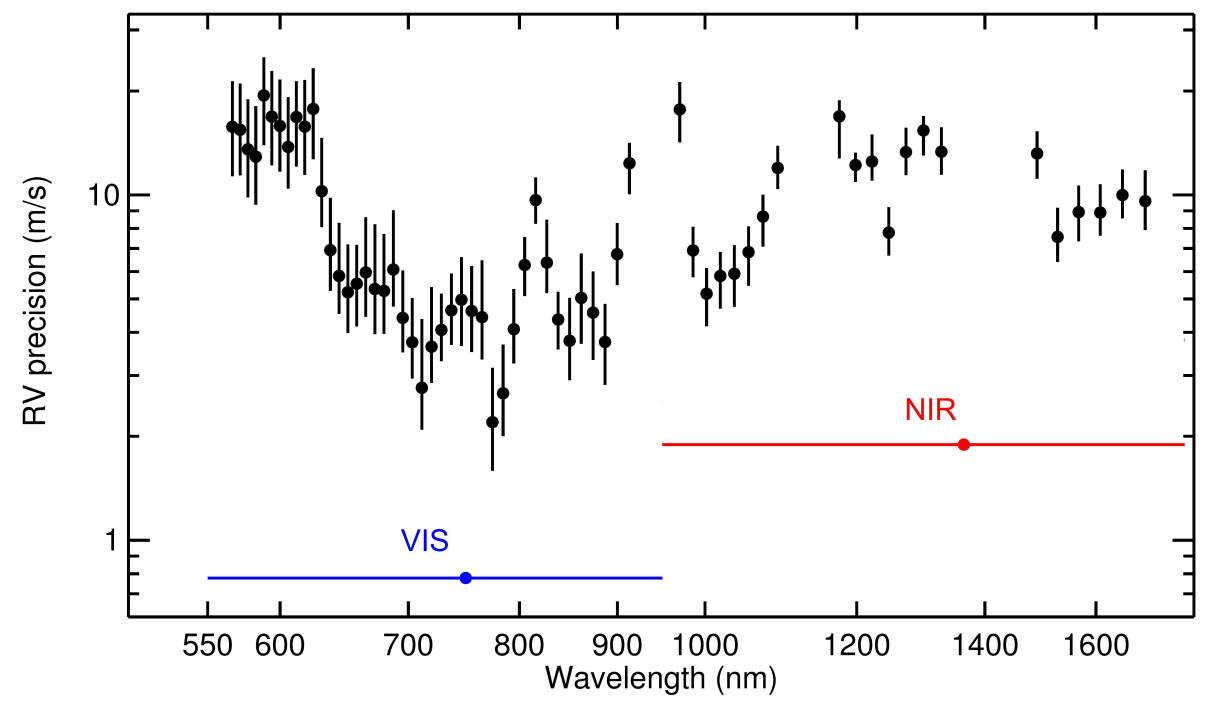

Figure 3. Relative internal radial velocity precision (representing SNR and spectral information content) for individual échelle orders, and integrated over the wavelength range of the visible-light and near-infrared spectrographs of CARMENES, for all M5 stars in the survey. In spite of the rather red colors of these stars, the wavelength range from $\sim 700 \mathrm{~nm}$ to $\sim 900 \mathrm{~nm}$ is the most favorable for precise radial velocity measurements. Adopted from Reiners et al. (2018b).

\subsection{The CARMENES Library of M Star Spectra}

The first publicly available data product from CARMENES is an atlas of $324 \mathrm{M}$ dwarf spectra, covering the wavelength range from 550 to $1700 \mathrm{~nm}$ (Reiners et al. 2018b). This data release includes the first large library of high-resolution near-infrared spectra of low-mass stars. One particularly interesting result in the context of Doppler planet surveys concerns the question about the best wavelength range for precise radial-velocity measurements of cool stars. Based on the expectation that this should be the near-infrared, several projects have been initiated to build near-IR Doppler spectrographs (see e.g. Fischer et al. 2016 and references therein). It is apparent from the CARMENES library, however, that state-of-the-art atmosphere models such as PHOENIX over-predict the depth of FeH bands (see Fig. 2). This, together with the fact that the TiO bands between 700 and $900 \mathrm{~nm}$ provide excellent RV information, shifts the balance from the region longward of $1 \mu \mathrm{m}$ into the range that is easily covered with CCD detectors.

The power of red-sensitive visible-light spectrographs for RV measurements of mid-M dwarfs is borne out by the CARMENES data. Figure 3 shows the internal precision achieved for the survey observations of M5 stars, separately for each spectrograph order, and (in color) integrated for each of the two spectrographs. Since this precision results from the SNR and the information content of the spectra, longer integrations would give better results, but all data points in the plot would move downward by the same factor. For even later spectral types, the near-infrared outperforms the red wavelength range, but there are not many stars with spectral types later than M5 that are bright enough for precise RV measurements with $4 \mathrm{~m}$ class telescopes and with reasonable integration times.

We have also observed a sample of stars covering the $\mathrm{O}$ to $\mathrm{K}$ spectral range; this library will be released soon (Caballero et al., in prep.).

\section{PLANETS IN THE CARMENES SURVEY}

\subsection{Planet Detections}

The CARMENES Survey sample includes a number of stars with previously known planets: GJ 15 A, GJ 436, GJ 176, GJ 536, GJ 1148, GJ 581, GJ 876, GJ 179, GJ 625, GJ 628, GJ 649, and GJ 849 (see Trifonov et al. 2018 and references therein). The first seven of these have planets with sufficiently short orbital periods to be 


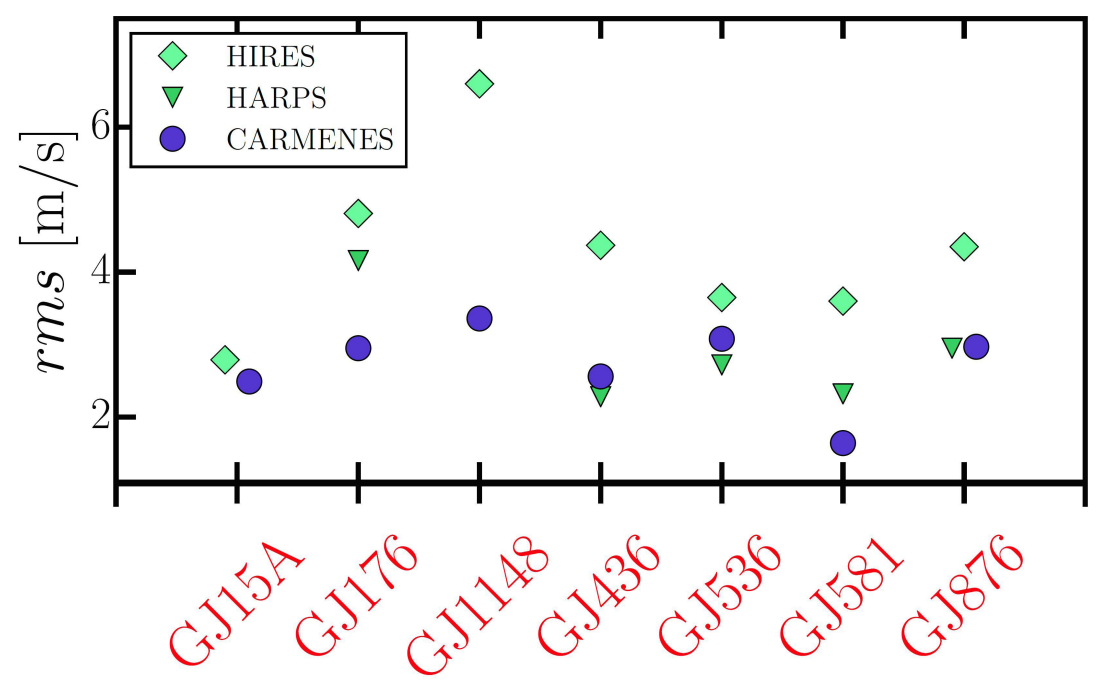

Figure 4. Weighted mean rms residual scatter around the best orbital fits for seven stars with planets, for HARPS, HIRES and CARMENES. The residuals of the CARMENES and HARPS data are very similar to each other, and lower when compared to HIRES. Adopted from Trifonov et al. (2018).

detectable with the first-year CARMENES observations. Trifonov et al. (2018) performed a combined analysis of the visible-arm CARMENES data with HIRES, HARPS, and HARPS-N data publicly available for these stars. In all cases except GJ $15 \mathrm{~A}$, they confirm the presence of the previously announced planets, and provide updated orbital fits for them.

The nearby bright M1 dwarf GJ 15 A shows clear radial-velocity variations with a period of $11.4 \mathrm{~d}$, which were observed with Keck/HIRES and interpreted as the signature of a planet (GJ 15 Ab) by Howard et al. (2014). However, the amplitude of this signal observed with HIRES, CARMENES, and HARPS-N (Pinamonti et al. 2018) appears to vary over time, suggesting that it might be due to stellar activity rather than a companion. While this question needs to be clarified with further data, the combined analysis of the HIRES and CARMENES by Trifonov et al. (2018) revealed a planet with a much longer orbital period (GJ 15 Ac), which was subsequently confirmed by Pinamonti et al. (2018).

The HIRES and CARMENES data also revealed a new outer planet in the GJ 1148 system. Based on the two combined data sets we derived a period of $P_{c}=533 \mathrm{~d}$, eccentricity $e_{c}=0.36$, and minimum mass $m_{c} \sin i=68 M_{\oplus}$ for GJ $1148 \mathrm{c}$.

The consistent joint analysis of HIRES, HARPS, and CARMENES data allows a direct comparison between these instruments. Figure 4 shows the residual rms scatter of the data points from the three spectrographs around the joint fits for seven stars. (No HARPS data are available for the Northern stars GJ 15 A and GJ 1148.) The CARMENES data have very similar or somewhat smaller scatter compared with the values from HARPS; this scatter is very likely indicative of the intrinsic radial-velocity "jitter" due to stellar activity. The rms of the HIRES data is generally somewhat higher.

A previously unknown Neptune-mass planet was found in the temperate zone of GJ617 A (HD 147379) in the visible-light CARMENES data (Reiners et al. 2018a). These data are shown in Fig. 5, phased to a period of 87.267 d. Archival data from HIRES support this discovery, although the planetary signal is not significant in the HIRES data alone. With a mass of $25 M_{\oplus}$, HD $147379 \mathrm{~b}$ occupies a mass range that is only sparsely populated around stars that are substantially less massive than the Sun. These objects are relatively easy to identify in the CARMENES data. The CARMENES Survey will therefore likely reveal a few additional such objects, and provide a good statistical assessment of their occurrence rate. 


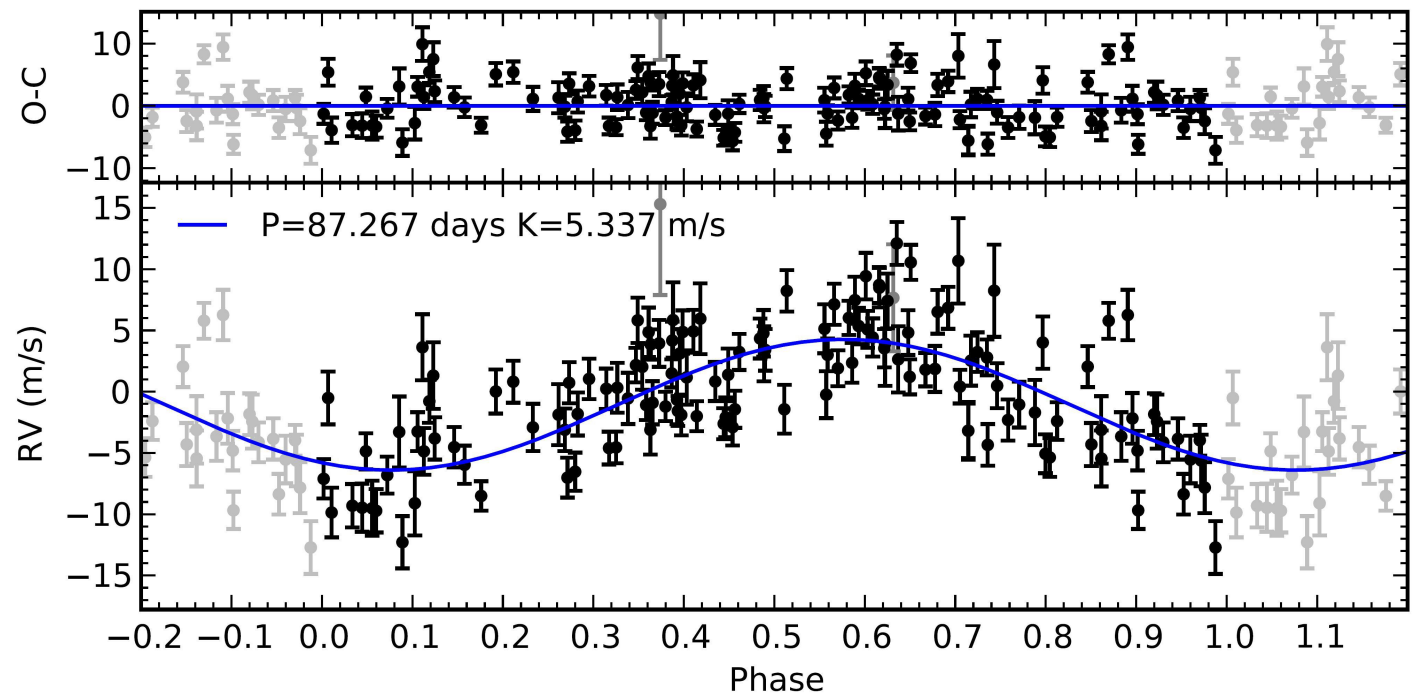

Figure 5. Radial velocities of GJ 617 A (HD 147379) from the CARMENES visible-light spectrograph, phased to a period of $87.267 \mathrm{~d}$.

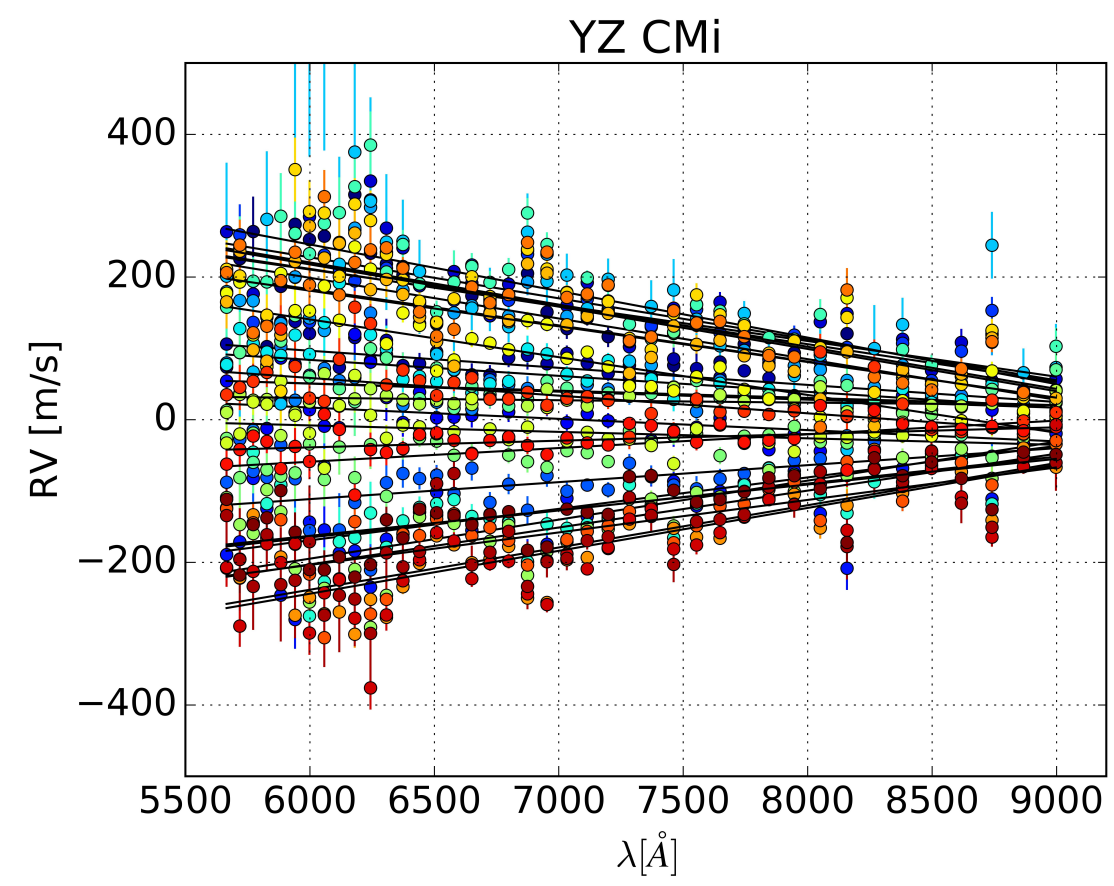

Figure 6. Wavelength-dependent radial velocities of the active star YZ CMi. For each observation, the RV was determined separately for each échelle order (set of points of a given color); these points were subsequently fitted with a straight line. The ensemble of lines thus represents the $\lambda$-dependent RV variations over time. For this star, it is apparent that the RV variations are much stronger at bluer wavelengths. This is a clear indication that the RV variations are caused by activity, not by a companion. 


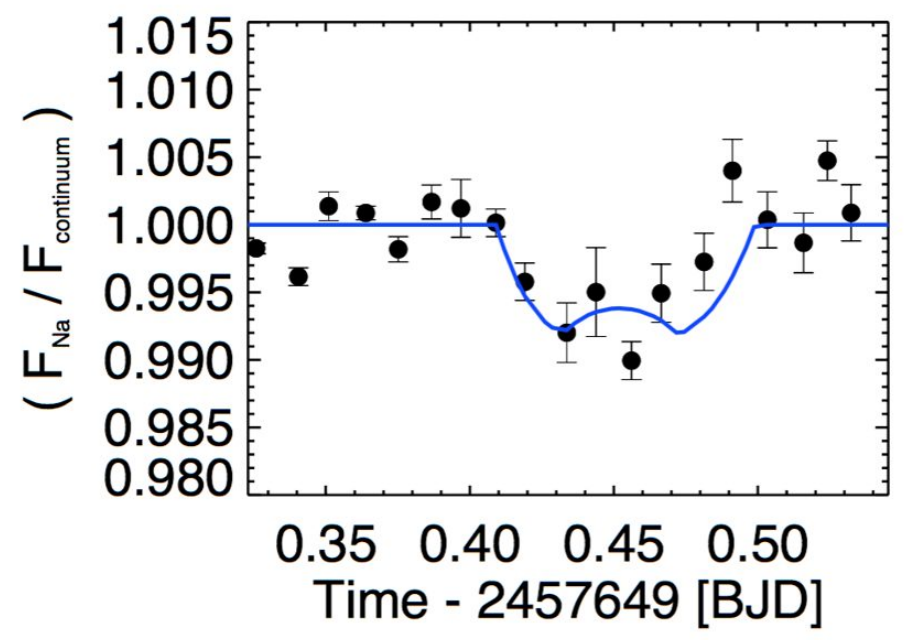

Figure 7. Differential light curve of the Na I D lines observed during a transit of the planet HD 189733 b. The graph shows the flux in the lines, divided by the flux in the nearby continuum, as a function of time. The excess sodium absorption indicates the presence of an extended planetary atmosphere. See text for more details.

\subsection{Distinguishing between Planets and Activity}

Radial velocities are measured from photospheric absorption lines. Variability of these lines may therefore introduce "noise" on precise RV measurements, and in the worst case mimic Keplerian signals, leading to false positives in planet searches. The ability to distinguish between such spurious signals and bona fide planet detections was the main motivation for the dual-spectrograph design of CARMENES, providing access to a large number of activity indicators, and enabling investigations of the observed radial velocity as a function of wavelength. The latter quantity is a standard product of the CARMENES data reduction pipeline: The RV is computed separately for each échelle order, and the slope of the RV vs. $\log \lambda$ determined by a linear fit, yielding the "chromatic index" (Zechmeister et al. 2018). The chromatic index is a powerful tool to distinguish between RV signals caused by planets and activity-induced signals: The motion of a star around the center of mass of the planetary system is strictly achromatic (i.e., chromatic index $=0$ within the measurement errors), whereas the signature of activity is usually stronger at shorter wavelengths, giving rise to non-zero chromatic indices. This is indeed the case for many of the active stars studied by Tal-Or et al. (2018), who also noted an (expected) tendency for an anti-correlation between RV and chromatic index. A beautiful example for this behavior is the strongly active star YZ CMi, shown in Fig. 6. Here the RV slopes clearly exclude a planetary origin of the RV variations, and they also contain valuable information on the active regions (temperature contrast and filling factor), which is difficult to obtain otherwise.

The pipeline also routinely produces line indices of activity indicators such as $\mathrm{H} \alpha$, the $\mathrm{Na}$ I $\mathrm{D}$ lines, and the Ca II infrared triplet for each observation. For many of the CARMENES target stars, periodograms of these quantities and of the chromatic index show peaks that are indicative of the stellar rotation period. If there is any radial-velocity signal at the same period or a harmonic, it can very likely also be attributed to rotationally modulated stellar activity rather than a planetary companion. In this way, the wide wavelength coverage of CARMENES, and the systematic analysis of multiple indicators of photospheric and chromospheric activity, help to avoid misinterpretations of the radial-velocity curves.

\subsection{Transiting Planets}

Within the scope of the CARMENES Survey, and with additional observing time obtained through independent "open time" proposals, we are also undertaking observations of known transiting planets. For K2-18 b, a lowmass planet orbiting an M2.5 dwarf in its temperate zone, we measured a mass of $\sim 9 M_{\oplus}$, implying a density of $\sim 4 \mathrm{~g} \mathrm{~cm}^{-3}$, which is compatible with a composition of $50 \% \mathrm{H}_{2} \mathrm{O}$ and $50 \% \mathrm{MgSiO}_{3}$ (Sarkis et al. 2018). This 


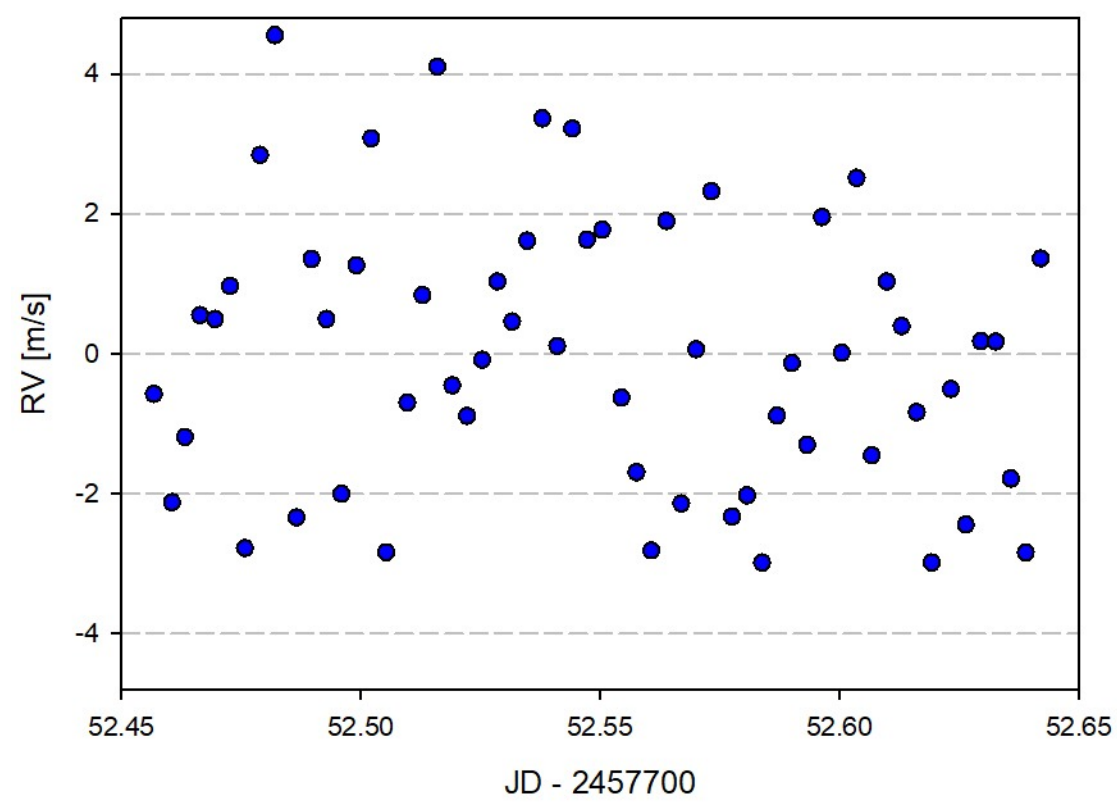

Figure 8. Radial velocities of Luyten's Star obtained with the CARMENES near-infrared spectrograph. The rms of this data set is $1.9 \mathrm{~m} \mathrm{~s}^{-1}$, demonstrating repeatability of the NIR measurements close to the photon noise limit.

mass measurement was very challenging due to the fact that the rotation period of the host star is rather close to the orbital period of the planet, necessitating a detailed analysis to disentangle the RV signal of the planet from activity-induced-variability, as described in the previous section.

We are also using CARMENES for observations of planets during transit, which can reveal information on the alignment of the planetary orbit with the stellar rotation axis through the Rossiter-McLaughlin effect, and on the composition of the planetary atmosphere. Figure 7 shows the differential light curve of the Na I D lines observed during a transit of the planet HD $189733 \mathrm{~b}$, i.e., the flux observed in these lines divided by the flux in the nearby continuum, normalized to the same quantity outside of transits. In the most naïve picture, the deeper transit means that the planet appears bigger at the Na wavelengths than in the continuum; this can be explained by $\mathrm{Na}$ absorption in an extended planetary atmosphere. However, one also has to take into account that the depth and shape of transit light curves is affected by limb darkening of the stellar disk, and that limb darkening in the stellar Na I D lines differs from continuum limb darkening. A model taking into account an extended planetary atmosphere and differential stellar limb darkening is shown along with the data in Fig. 7 . Transit data of additional atomic and molecular lines in HD 189733 and other stars are currently under analysis.

\subsection{Radial Velocity Performance}

During the early phases of CARMENES operations, a number of instrumental effects and calibration issues were identified, which affect the data from the visible-light arm on the $\mathrm{m} \mathrm{s}^{-1}$ level. These include incorrect settings of the atmospheric dispersion corrector (ADC) angles, affecting the illumination of the fibers in the front end mainly at larger zenith angles, improper illumination of the calibration fibers by the Fabry-Pérot etalons, and a thermal drift of the etalons after switching on the white light lamps used to illuminate them.

Therefore, we calculated for each night dedicated to the CARMENES Survey an instrumental nightly zero point (NZP) of the RVs by using all the stars with small RV variability (RV-quiet stars) observed in that night (see Trifonov et al. 2018). We then corrected each RV measurement for its NZP and propagated the NZP error into the measurement uncertainty. The median NZP uncertainty was found to be $\sim 1 \mathrm{~m} \mathrm{~s}^{-1}$, while their scatter is $\sim 2.5 \mathrm{~m} \mathrm{~s}^{-1}$. For the seven planetary systems investigated by Trifonov et al. (2018), we found the NZP-corrected 

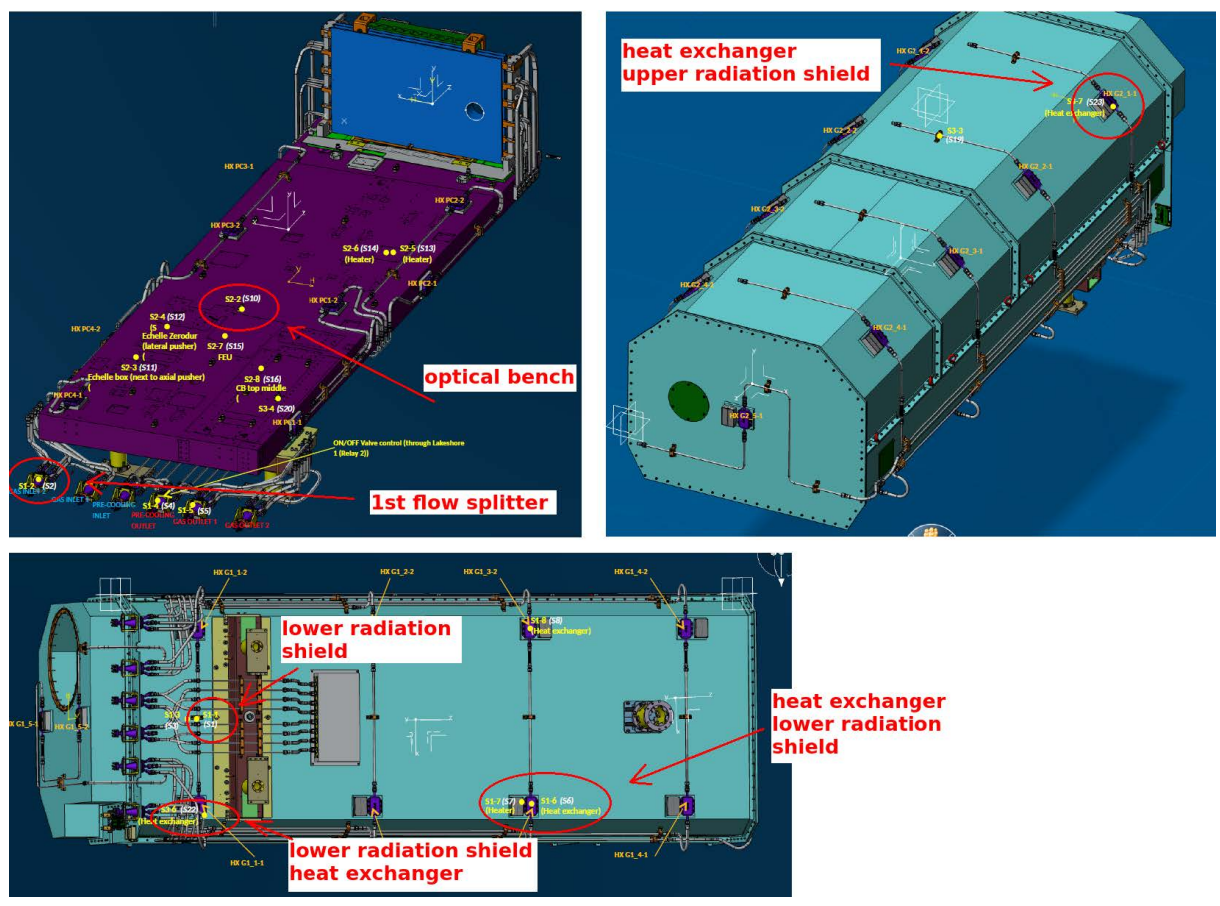

Figure 9. Distribution of temperature sensors in the CARMENES near-infrared spectrograph.

RVs to improve the rms velocity dispersion around the best-fit models by $\sim 25 \%$ on average. With an improving understanding of the spectrograph and coupling optics, we expect the calibration procedure to yield better RV data than the NZP correction scheme. We are currently investigating whether this is already the case after correcting the problems mentioned above, but assessing the level of instrumental errors is complicated by the fact that the program stars show an intrinsic RV scatter with a typical amplitude of $\sim 3 \mathrm{~ms}^{-1}$. In any case, our ability to detect small planets of $\mathrm{M}$ dwarfs is limited by that scatter rather than instrumental precision.

In the long run, we are planning to bring the single-measurement instrumental errors in the visible channel down to well below the $1 \mathrm{~m} \mathrm{~s}^{-1}$ level. This will require a better understanding (and perhaps improvement) of the thermal behavior of the spectrograph, which follows the temperature of the surrounding chamber with a long time constant. We are therefore developing a detailed optical model of the spectrograph, which is better suited for investigating the effects of mechanical drifts on the derived RVs than commercial ray tracing packages (Tala Pinto et al. 2018). We are also scrutinizing the end-to-end chain of observations, calibration, and data reduction for additional sources of error that may still be lurking below the level of stellar jitter. While improvements along these lines will have little impact on the main CARMENES Survey of M stars, they will enable additional science projects targeting stars with very quiet atmospheres.

The CARMENES near-infrared arm is also providing high-quality data, although its characterization is lagging somewhat behind that of its visible-light counterpart. In Fig. 8 we show high-cadence observations of Luyten's star with an RV rms of $1.9 \mathrm{~m} \mathrm{~s}^{-1}$ derived exclusively form the near-IR data. From this short time series alone one can deduce that many important contributors to the RV error budget are well below the $2 \mathrm{~m} \mathrm{~s}^{-1}$ level. With appropriate observing strategies, operational procedures, and calibration methods, it should be possible to also bring the long-term stability into this range.

Among the limiting factors in achieving stable operations is the thermal management of the actively cooled near-infrared spectrograph, which is much more challenging than that of its visible-light counterpart, which follows the ambient temperature passively. For example, daily exchanges of the liquid nitrogen bottles or slow loss of the vacuum isolating the cooling lines affect the thermal stability of the near-IR spectrograph. The temperature inside the near-IR vacuum tank is monitored at various locations (see Fig. 9), and correlating this information with external events has led to insight into the thermal behavior that has been invaluable for 


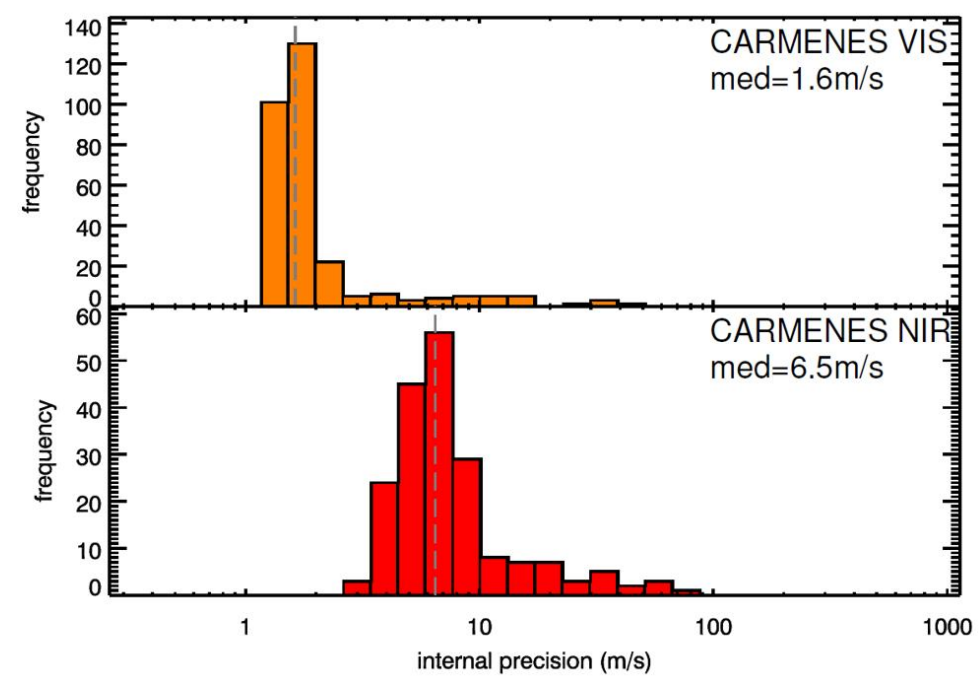

Figure 10. Internal precision of radial velocities from the CARMENES Survey observations, for the visible-light (top) and near-infrared (bottom) channels. These histograms show the photon noise limit of the data, i.e., they reflect the SNR and information content of the spectra, not the calibration or stability of the spectrographs.

improving the stability. In addition, we have over time been able to optimize the control law of the temperature control system, which also had a profound impact on the RV stability. We are still aware of several weaknesses, which we hope to be able to address with small future hardware upgrades.

It should be pointed out, however, that the near-infrared radial-velocities from the CARMENES Survey will only in exceptional cases reach the precision of those from the visible-light arm, simply because most of the RV information is at wavelengths shorter than $0.96 \mu \mathrm{m}$, as discussed in Sect. 4.4. This is further illustrated in Fig. 10, which shows a histogram of the internal precision achieved in the CARMENES Survey observations for both channels. As the internal precision is essentially a measure of the signal-to-noise and spectral information content, it sets a lower limit to the achievable radial-velocity precision, irrespective of spectrograph stability or calibration errors. The distribution in Fig. 10 thus reflects the observing strategy adopted for the CARMENES Survey: Exposures are stopped after an integration time that is sufficient to achieve a photon noise corresponding to 1 to $2 \mathrm{~m} \mathrm{~s}^{-1}$, which is well below the typical stellar activity level of 3 to $4 \mathrm{~ms}^{-1}$. This exposure time is too short to get to the $\sim 2 \mathrm{~m} \mathrm{~s}^{-1}$ level in the near-IR arm, for almost all of the stars in our survey sample. This result does not preclude the use of near-IR spectrographs for high-precision radial-velocity measurements, but it demonstrates that comparatively long integration times are needed.

\section{FUTURE PERSPECTIVES FOR CARMENES}

CARMENES is currently used during most nights for the dedicated $\mathrm{M}$ star survey, but the instrument is also under heavy demand in "open time" for other scientific topics. It will certainly remain a powerful facility for many years to come, after its primary mission has been completed. One can foresee many uses of CARMENES as a "general purpose" tool for stellar astrophysics, but the topics discussed above - planet surveys, transit follow-up, planetary atmospheres - will likely remain at the forefront.

The demand for observing time with RV instruments on $4 \mathrm{~m}$ class telescopes is expected to rise sharply when the first data from the TESS mission (Ricker et al. 2014), which was launched in April 2018, become available. TESS will find planets transiting relatively bright stars of all spectral types. Working in the red and nearinfrared, CARMENES is particularly suited for follow-up of the coolest of these stars. Furthermore, concurrent observations with TESS and CARMENES can open a new view on stellar activity through simultaneous precise photometric and wide-band spectroscopic time series. The anticipated wealth of TESS discoveries will provide promising targets for many years of CARMENES operations, leading into the era of the European PLATO mission (Rauer et al. 2014), which will take transit surveys to the next level in the middle of the coming decade. 


\section{ACKNOWLEDGMENTS}

CARMENES is an instrument for the Centro Astronómico Hispano-Alemán de Calar Alto (CAHA, Almería, Spain). CARMENES is funded by the German Max-Planck-Gesellschaft (MPG), the Spanish Consejo Superior de Investigaciones Científicas (CSIC), the European Union through FEDER/ERF FICTS-2011-02 funds, and the members of the CARMENES Consortium (Max-Planck-Institut für Astronomie, Instituto de Astrofísica de Andalucía, Landessternwarte Königstuhl, Institut de Ciències de l'Espai, Insitut für Astrophysik Göttingen, Universidad Complutense de Madrid, Thüringer Landessternwarte Tautenburg, Instituto de Astrofísica de Canarias, Hamburger Sternwarte, Centro de Astrobiología and Centro Astronómico Hispano-Alemán), with additional contributions by the Spanish Ministry of Science, the German Science Foundation through the Major Research Instrumentation Program and DFG Research Unit FOR2544 "Blue Planets around Red Stars", the Klaus Tschira Stiftung, the states of Baden-Württemberg and Niedersachsen, and by the Junta de Andalucía.

\section{REFERENCES}

[1] Alonso-Floriano, F.J., Morales, J.C., Caballero, J.A., Montes, D., Klutsch, A., et al. (2015). CARMENES input catalogue of $M$ dwarfs. I. Low-resolution spectroscopy with CAFOS. A\&A 577, A128

[2] Amado, P.J., Lenzen, R., Cardenas, M.C., Sánchez-Blanco, E., Becerril, S., et al. (2012). CARMENES. V: non-cryogenic solutions for YJH-band NIR instruments. In Modern technologies in space- and ground-based telescopes and instrumentation II. Eds. Navarro, R., Cunningham, C.R., \& Prieto, E., SPIE 84501U

[3] Bauer, F.F., Zechmeister, M., \& Reiners, A. (2015). Calibrating echelle spectrographs with Fabry-Pérot etalons. A\&A 581, A117

[4] Becerril, S., Lizon, J.L., Sánchez-Carrasco, M.A., Mirabet, E., Amado, P., et al. (2012). CARMENES. III: an innovative and challenging cooling system for an ultrastable NIR spectrograph. In Modern technologies in space- and ground-based telescopes and instrumentation II. Eds. Navarro, R., Cunningham, C.R., \& Prieto, E., SPIE $84504 \mathrm{~L}$

[5] Caballero, J.A., Cortés-Contreras, M., Alonso-Floriano, F.J., Montes, D., Quirrenbach, A., et al. (2016a). Carmencita, The CARMENES Input Catalogue of Bright, Nearby M Dwarfs. In The 19th Cambridge Workshop on Cool Stars, Stellar Systems, and the Sun. Id 148

[6] Caballero, J.A., Guàrdia, J., López del Fresno, M., Zechmeister, M., de Juan, E., et al. (2016b). CARMENES: data flow. In Observatory operations: strategies, processes, and systems VI. Eds. Peck, A.B., Seaman, R.L., \& Benn, C.R., SPIE 99100E

[7] Cortés-Contreras, M., Béjar, V.J.S., Caballero, J.A., Gauza, B., Montes, D., et al. (2017). CARMENES input catalogue of $M$ dwarfs. II. High-resolution imaging with FastCam. A\&A 597, A47

[8] Fischer, D.A., Anglada-Escudé, G., Arriagada, P., Baluev, R.V., Bean, J.L., et al. (2016). State of the field: extreme precision radial velocities. PASP 128, 066001

[9] Fuhrmeister, B., Czesla, S., Schmitt, J.H.M.M., Jeffers, S.V., Caballero, J.A., et al. (2018). The CARMENES search for exoplanets around $M$ dwarfs: Wing asymmetries of $H \alpha, N a I D$, and HeI lines. A\&A, in press (arXiv:1801.10372)

[10] Garcia-Piquer, A., Morales, J.C., Ribas, I., Colomé, J., Guàrdia, J., et al. (2017). Efficient scheduling of astronomical observations. Application to the CARMENES radial-velocity survey. A\&A 604, A87

[11] Helmling, J., Wagner, K., Hernández Castaño, L., Benítez, D., Marín Molina, J., et al. (2016). CARMENES: Interlocks or the importance of process visualization and system diagnostics in complex astronomical instruments. In Ground-based and Airborne Instrumentation for Astronomy VI. Eds. Evans, C.J., Simard, L., \& Takami, H., SPIE 990890

[12] Howard, A.W., Marcy, G.W., Fischer, D.A., Isaacson, H., Muirhead, P.S. (2014). The NASA-UC-UH ETAEarth program. IV. A Low-mass planet orbiting an M dwarf 3.6 pc from Earth. ApJ 794, 51

[13] Husser, T.O., Wende-von Berg, S., Dreizler, S., Homeier, D., Reiners, A., et al. (2013). A new extensive library of PHOENIX stellar atmospheres and synthetic spectra. A\&A 553, A6

[14] Jeffers, S.V., Schoefer, P., Lamert, A., Reiners, A., Montes, D., et al. (2018). CARMENES input catalogue of $M$ dwarfs: III. Rotation and activity from high-resolution spectroscopic observations. A\&A 614, A76 
[15] Montes, D., González-Peinado, R., Tabernero, H.M., Caballero, J.A., Marfil, E., et al. (2018). Calibrating the metallicity of $M$ dwarfs in wide physical binaries with $F-, G$-, and $K$ - primaries - I: Highresolution spectroscopy with HERMES: stellar parameters, abundances, and kinematics. MNRAS, in press (arXiv:1805.05394)

[16] Passegger, V.M., Reiners, A., Jeffers, S.V., Wende-von Berg, S., Schoefer, P., et al. (2018). The CARMENES search for exoplanets around $M$ dwarfs - Photospheric parameters of target stars from high-resolution spectroscopy. A\&A 615, A6

[17] Pinamonti, M., Damasso, M., Marzari, F., Sozzetti, A., Desidera, S., et al. (2018). The HADES RV Programme with HARPS-N@TNG VIII. Gl15A: A multiple wide planetary system sculpted by binary interaction. A\&A, in press (arXiv:1804.03476)

[18] Quirrenbach, A., Amado, P.J., Mandel, H., Caballero, J.A., Mundt, R., et al. (2010). CARMENES: Calar Alto high-Resolution search for $M$ dwarfs with Exo-earths with Near-infrared and optical Echelle Spectrographs. In Ground-based and airborne instrumentation for astronomy III. Eds. McLean, I.S., Ramsay, S.K., \& Takami, H., SPIE 773513

[19] Quirrenbach, A., Amado, P.J., Seifert, W., Sánchez Carrasco, M.A., Mandel, H., et al. (2012). CARMENES. I: instrument and survey overview. In Ground-based and airborne instrumentation for astronomy IV. Eds. McLean, I.S., Ramsay, S.K., \& Takami, H., SPIE 84460R

[20] Quirrenbach, A., Amado, P.J., Caballero, J.A., Mundt, R., Reiners, A., et al. (2014). CARMENES instrument overview. In Ground-based and airborne instrumentation for astronomy V. Eds. Ramsay, S.K., McLean, I.S., \& Takami, H., SPIE 91471F

[21] Quirrenbach, A., Amado, P.J., Caballero, J.A., Mundt, R., Reiners, A., et al. (2016). CARMENES: an overview six months after first light. In Ground-based and airborne instrumentation for astronomy VI. Eds. Evans, C.J., Simard, L., \& Takami, H., SPIE Vol. 9908

[22] Rauer, H., Catala, C., Aerts, C., Appourchaux, T., Benz, W., et al. (2014). The PLATO 2.0 mission. Exp. Astron. 38, 249

[23] Reiners, A., Ribas, I., Zechmeister, M., Caballero, J.A., Trifonov, T., et al. (2018a). The CARMENES search for exoplanets around $M$ dwarfs. HD147379 b: A nearby Neptune in the temperate zone of an early-M dwarf. A\&A 609, L5

[24] Reiners, A., Zechmeister, M., Caballero, J.A., Ribas, I., Morales, J.C., et al. (2018b). The CARMENES search for exoplanets around $M$ dwarfs. High-resolution optical and near-infrared spectroscopy of 324 survey stars. A\&A 612, A49

[25] Ricker, G.R., Winn, J.N., Vanderspek, R., Latham, D.W., Bakos, G.Á., et al. (2014). Transiting Exoplanet Survey Satellite. JATIS 1, 014003

[26] Sarkis, P., Henning, T., Kürster, M., Trifonov, T., Zechmeister, M., et al. (2018). The CARMENES search for exoplanets around $M$ dwarfs: A low-mass planet in the temperate zone of the nearby K2-18. AJ 155, 257

[27] Sarmiento, L.F., Reiners, A., Seemann, U., Lemke, U., Winkler, J., et al. (2014). Characterizing U-Ne hollow cathode lamps at near-IR wavelengths for the CARMENES survey. In Ground-based and airborne instrumentation for astronomy V. Eds. Ramsay, S.K., McLean, I.S., \& Takami, H., SPIE 914754

[28] Schäfer, S., Guenther, E.W., Reiners, A., Winkler, J., Pluto, M., \& Schiller, J. (2018). Two Fabry-Pérots and two calibration units for CARMENES. These proceedings, 10702-262

[29] Seifert, W., Sánchez-Carrasco, M., Xu, W., Cárdenas, C., Sánchez-Blanco, E., et al. (2012). CARMENES. II: optical and opto-mechanical design. In Ground-based and airborne instrumentation for astronomy IV. Eds. McLean, I.S., Ramsay, S.K., \& Takami, H., SPIE 844633

[30] Seifert, W., Xu, W., Stahl, O., Hagen, H.J., Sánchez Carrasco, M.A., et al. (2016). CARMENES: the VIS channel spectrograph in operation. In Ground-based and Airborne Instrumentation for Astronomy VI. Eds. Evans, C.J., Simard, L., \& Takami, H., SPIE 990865

[31] Stürmer, J., Stahl, O., Schwab C., Seifert, W., Quirrenbach, A., et al. (2014). CARMENES in SPIE 2014. Building a fibre link for CARMENES. In Advances in optical and mechanical technologies for telescopes and instrumentation. Eds. Navarro, R., Cunningham, C.R., \& Barto, A.A., SPIE 915152 
[32] Tal-Or, L., Zechmeister, M., Reiners, A., Jeffers, S.V., Schöfer, P., et al. (2018). The CARMENES search for exoplanets around $M$ dwarfs: Radial-velocity variations of active stars in visual-channel spectra. A\&A 614, A122

[33] Tala Pinto, M., Chanumolu, A., Quirrenbach, A., Reffert, S., Zechmeister, M., \& Bauer, F. (2018). Physical modeling of echelle spectrographs: the CARMENES case study. These proceedings, 10705-68

[34] Trifonov, T., Kürster, M., Zechmeister, M., Tal-Or, L., Caballero, J.A., Quirrenbach, A., et al. (2018). The CARMENES search for exoplanets around $M$ dwarfs . First visual-channel radial-velocity measurements and orbital parameter updates of seven M-dwarf planetary systems. A\&A 609, A117

[35] Zechmeister, M., Anglada-Escudé, G., \& Reiners, A. (2014). Flat-relative optimal extraction. A quick and efficient algorithm for stabilised spectrographs. A\&A 561, A59

[36] Zechmeister, M., Reiners, A., Amado, P.J., Azzaro, M., Bauer, F.F., et al. (2018). Spectrum radial velocity analyser (SERVAL). High-precision radial velocities and two alternative spectral indicators. A\&A 609, A12 